\title{
Study on the properties of Rayleigh channel wave in mine gob area with $3 D$ finite-difference method
}

\author{
Yuge Jia ${ }^{1}$ Wenqiang Yang ${ }^{3}$ Dechun $\mathrm{Li}^{4}$ \\ School of Resource and Earth Science \\ China University of Mining and Technology \\ Xuzhou 221008, China \\ gegecumt@126.com
}

\author{
Jiulong Cheng ${ }^{2}$ \\ College of Geoscience and Surveying Engineering \\ China University of Mining and Technology \\ Beijing 100083, China
}

\begin{abstract}
Underground mine gob area is a kind of potential geological disasters, this paper studies the formation and propagation of the Rayleigh-channel wave under the condition of existing mine gob area, using three-dimensional inhomogeneous isotropic medium wave equation and solving the elastic wave equation with finite- difference method. According to the seismological features of the mine gob area, we design several simple models of the mine gob area. Finally we analyze the characters of Rayleigh-channel wave under the condition of existing mine gob area and provide a theoretical basis for the channel wave exploration.
\end{abstract}

Key words-Rayleigh-channel wave; finite-difference method; numerical simulation; mine gob area

\section{INTRODUCTION}

The mined gob area is caused by coal mining underground. They may be filled with gas, water or silt, etc. With the passage of time, the surrounding geological structure around the cavity will changes and result in changes of geophysical characteristics of mined out areas.

Regardless of what it is filled underground mining empty area has an obvious reflection interface of wave impedance in the edge of it. There exist a significant velocity differences between the empty area media and surrounding rock.
Rayleigh-channel wave exploration underground is to detect mined out area underground using this physical prerequisite.

When the seismic wave is excited in the low velocity layer, the velocity of surrounding rock is relatively high. When the incident angle is greater than critical angle, it will totally reflect at the top and bottom interface. Totally reflection will reverberate and superimpose mutually with refraction. The low coal seam is like a waveguide layer and it is known as channel wave.

When Rayleigh-channel wave propagate, the particles move in the vertical plane along the direction of the wave propagation and the movement is a reverse ellipse.

In this paper, we designed a few simple geological model of the mined out area. The finite-difference method is used to simulate the Rayleigh-channel wave propagating in the three dimensional space.

\section{FINITE DIFFERENCE NUMERICAL SOLUTION OF WAVE} EQUATION

\section{A. The Inhomogeneous Is Otropic Elastic Wave Equation}

\section{Satisfied By Channel Wave}

Equation (1) is for the inhomogeneous elastic wave with the isotropic media ${ }^{[1,2,3,4]}$.

$\frac{\partial^{2} u_{x}}{\partial t^{2}}=\left(\frac{\lambda+2 \mu}{\rho}\right)\left[\frac{\partial^{2} u_{x}}{\partial x^{2}}+\frac{\partial^{2} u_{y}}{\partial x \partial y}+\frac{\partial^{2} u_{z}}{\partial x \partial z}\right]+\left(\frac{\mu}{\rho}\right)\left[\frac{\partial^{2} u_{x}}{\partial y^{2}}+\frac{\partial^{2} u_{x}}{\partial z^{2}}-\frac{\partial^{2} u_{y}}{\partial x \partial y}-\frac{\partial^{2} u_{z}}{\partial x \partial z}\right]$
$\frac{\partial^{2} u_{y}}{\partial t^{\dagger}}=\left(\frac{\lambda+2 \mu}{\rho}\right)\left[\frac{\partial^{2} u_{x}}{\partial x \partial z}+\frac{\partial^{2} u_{y}}{\partial y^{2}}+\frac{\partial^{2} u_{z}}{\partial y \partial z}\right]+\left(\frac{\mu}{\rho}\right)\left[\frac{\partial^{2} u_{y}}{\partial x^{2}}+\frac{\partial^{2} u_{y}}{\partial z^{2}}-\frac{\partial^{2} u_{x}}{\partial x \partial y}-\frac{\partial^{2} u_{z}}{\partial y \partial z}\right](1)$
$\frac{\partial^{2} u_{x}}{\partial t^{2}}=\left(\frac{\lambda+2 \mu}{\rho}\right)\left[\frac{\partial^{2} u_{x}}{\partial x \partial z}+\frac{\partial^{2} u_{y}}{\partial y \partial z}+\frac{\partial^{2} u_{z}}{\partial z^{2}}\right]+\left(\frac{\mu}{\rho}\right)\left[\frac{\partial^{2} u_{z}}{\partial x^{2}}+\frac{\partial^{2} u_{z}}{\partial y^{2}}-\frac{\partial^{2} u_{x}}{\partial x \partial z}-\frac{\partial^{2} u_{y}}{\partial y \partial z}\right]$ 


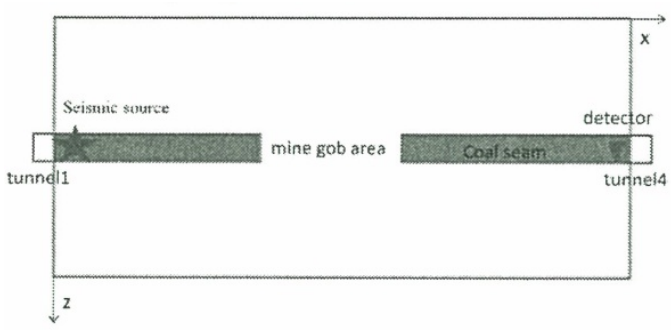

Fig. 1. Profile of geological model

TABLE I. ELASTIC PARAMETERS OF GEOLOGICAL MODEL

\begin{tabular}{|l|c|c|c|}
\hline & $V_{p}(\mathrm{~m} / \mathrm{s})$ & $V_{s}(\mathrm{~m} / \mathrm{s})$ & $\rho\left(\mathrm{kg} / \mathrm{m}^{3}\right)$ \\
\hline 1 Roof & 2600 & 1800 & 2500 \\
\hline 2Coal seam & 2000 & 1200 & 1400 \\
\hline 3 Bottom & 2600 & 1800 & 2500 \\
\hline
\end{tabular}

In witch, $\lambda$ and $\mu$ are Lame coefficient. $u_{x}, u_{y}$ and $u_{z}$ are components of displacement.

As shown in Fig.1, when the explosive source is excited, along $\mathrm{X}$-direction, $\mathrm{X}$ component mainly indicates Rayleigh-channel wave. Along y-direction, Y component mainly indicates Rayleigh-channel wave.

\section{B. Geological model and parameters}

In this paper, we solve the Equation (1) using Finite-difference method. Profile of geological model is shown in Fig.1.

Uniform grid is $1 \mathrm{~m} \times 1 \mathrm{~m} \times 1 \mathrm{~m}$ and model scale is $M=200 \Delta x \times 100 \Delta y \times 100 \Delta z$. The main frequency of Ricker wavelet is $100 \mathrm{~Hz}$ and the time sampling interval is $\Delta t=0.0002 \mathrm{~s}$. Seismic source locates at $x=10 \mathrm{~m}, y=50 \mathrm{~m}$, $\mathrm{z}=50 \mathrm{~m}$.

\section{NUMERICAL SIMULATION OF RAYLEIGH-CHANNEL}

WAVE FIELD UNDER THE CONDITION OF MINE GOB AREA

\section{A. Geologic Model}

The thickness of coal seam is $4 \mathrm{~m}$. Along $\mathrm{x}$-direction, the mine gob area range from $60 \mathrm{~m}$ to $80 \mathrm{~m}$, along z-direction it same with the thickness of the coal seam, ranging from the $48 \mathrm{~m}$ to the $52 \mathrm{~m}$, and along $\mathrm{y}$-direction it is infinite.

In this paper, we calculated three simple models of mine gob area and then compare the results with the results of the uniform coal seam model.

B. Wave Field Snapshot Across $x-y$ Profile $(z=50 m$,

$$
t=0.1 \mathrm{~s} \text { ) }
$$

The energy of vertical component $\mathrm{Z}$ is basically leaked to the surrounding rock and the vertical component $Z$ energy is very weak. Therefore, this paper only studies the propagation law of the horizontal component $\mathrm{X}$ of Rayleigh-channel wave.
1)For air $v_{p}=340 \mathrm{~m} / \mathrm{s}$ and $v_{s}=0 \mathrm{~m} / \mathrm{s}$.

2) For water $v_{p}=1500 \mathrm{~m} / \mathrm{s}$ and $v_{s}=0 \mathrm{~m} / \mathrm{s}$.

3)For silt $v_{p}=1800 \mathrm{~m} / \mathrm{s}$ and $v_{s}=150 \mathrm{~m} / \mathrm{s}$.

Wave field snapshot of $\mathrm{X}$ component for four models are as shown in Fig.2.Comparison the wave field snapshot across $x-y$ profile:

- When the coal seam is uniform, it can form the steady Rayleigh-channel wave. Along x-direction, with the increase of propagation time and distance, energy decrease and the numbers of wave crests and troughs increase.

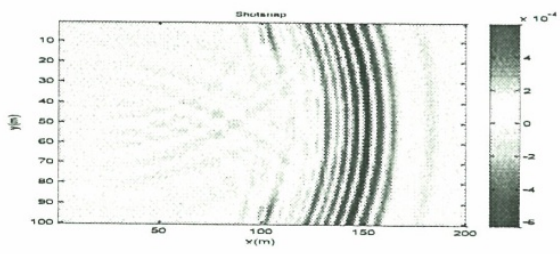

(a) Uniform coal seam model

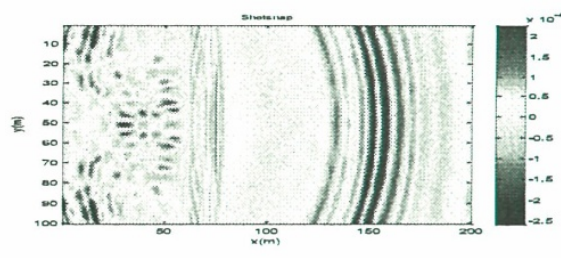

(b) Mine gob area model with air

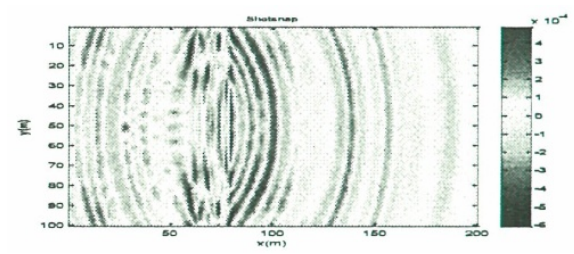

(c) Mine gob area model with water

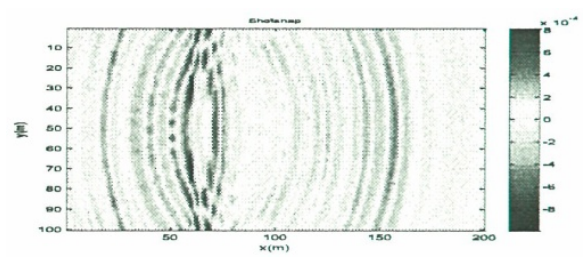

(d) Mine gob area model with silt

Fig. 2. Wave field snapshot

- When the mine gob area is filled with air, on the right side of the mine gob area the transmission channel wave is newly formed and on the left side reflected channel wave is newly formed.

- $\quad$ The transmission and reflection channel wave energy is about $1 / 3$ times of the channel wave energy in the case of uniform coal seam.

- When filled with water, the strong reflection channel wave is formed on the left side of the mine gob area. The transmission and reflection channel wave energy is 
about $1 / 2$ times of the channel wave energy in the case of uniform coal seam.

- When filled with silt, the reflection channel wave formed on the left side is stronger.

C. Wave Field Snapshot Across $x-z$ Profile $(y=50 m$, $t=0.1 s$ )

As shown in Fig.3. Comparison the wave field snapshot across $x-z$ profile:

- In the case of uniform coal seam, it forms a stable strong channel wave in coal seam.

- In the case of mine gob area, it forms transmission channel wave and reflection channel wave. The more energy leaks into the surrounding rock.

- When filled with air, the energy attenuation is the most serious.

- When filled with water or silt, the reflection channel wave is strong and the transmission channel wave energy is weak.

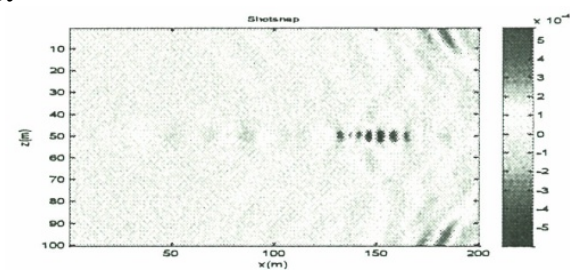

(a) Uniform coal seam model

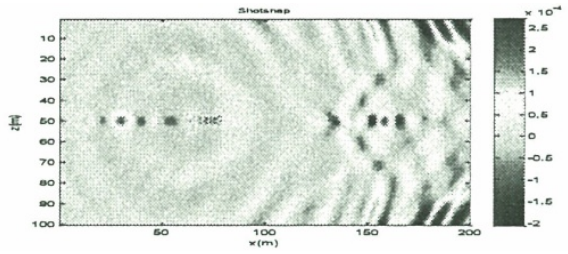

(b) Mine gob area model with air

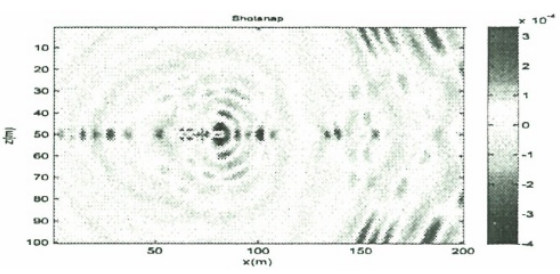

(c) Mine gob area model with water

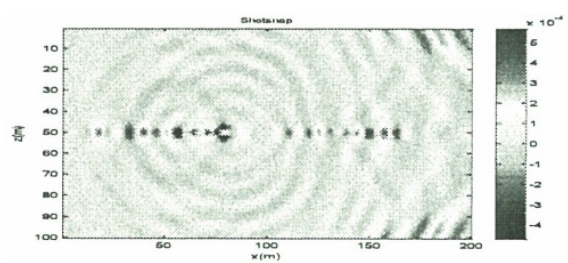

(d) Mine gob area model with silt

Fig. 3. Wave field snapshot
D. wave field record section along tunnel $4(x=190 \mathrm{~m}$, $y=50 \mathrm{~m}$ )

The explosion source is exited in tunnel 1 and wave field is recorded along tunnel 4 .

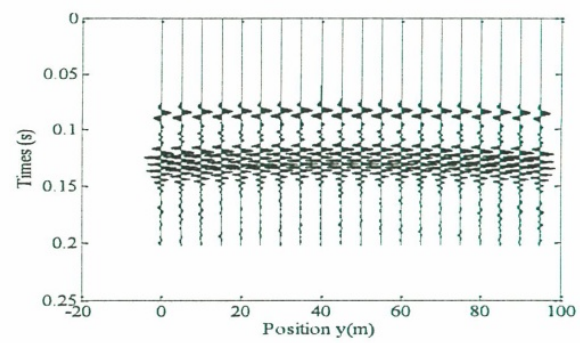

(a) Uniform coal seam model

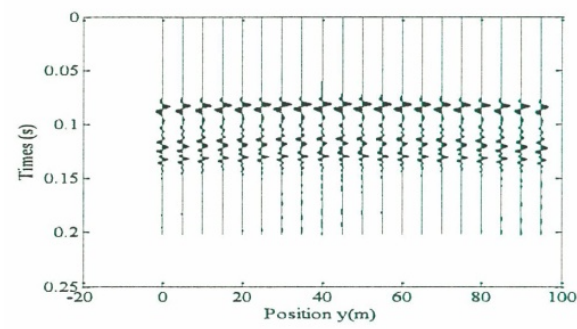

(b) Mine gob area model with air

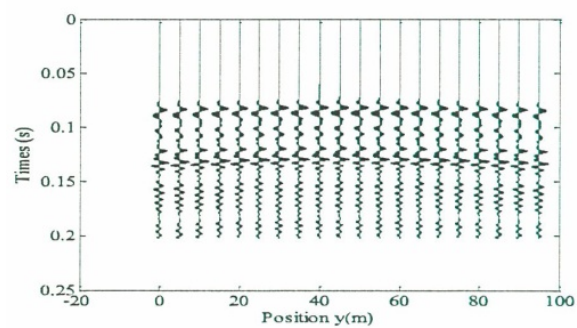

(c) Mine gob area model with water

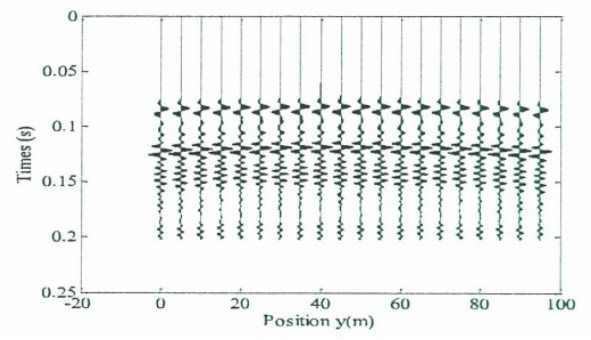

(d) Mine gob area model with silt

Fig. 4. Wave field record section 
In the case of uniform coal seam, it records direct waves and channel wave. In the case of mine gob area, it records two direct waves and transmission channel wave. Four record profiles corresponding 4 cases are shown in Fig.4. In order to clearly identify channel wave recorded, records are amplified 10000 times.

Comparing of record profiles:

- In the case of uniform coal seam, channel wave recorded is stable and very strong.

- When filled with air, the energy attenuation is the most serious.

- When filled with water and silt, especially in Fig.4 (d), we can see two direct waves and transmission channel wave.

\section{CONCLUSIONS}

This paper demonstrates that the mine gob area and its filling material have effects on the formation and propagation of the Rayleigh-channel wave field:

- When the channel wave goes through the mine gob area, changing in the propagation conditions may result in being reflected and converted many times in the mine gob area and then once again into the coal seam to form transmission and reflected channel wave .

- In this paper we only designed simple models and the actual codition is more complex.
In a word, it is urgent to study the formation and propagation of the channel wave in the case of complex small structures, which provides a theoretical basis for the practical exploration.

\section{ACKNOWLEDGEMENTS:}

This work was funded by the National Natural Science Fund (NNSF, Project: 41304)

\section{REFERENCE}

[1] Yang S T, Cheng J L. Numerical simulation of fore detecting with seismic in coal roadway and study of wave field characteristics. Journal of China Coal Society(in Chinese)[J], 2010, 35(10): $1633 \sim 1637$.

[2] G Z, Cheng J Y, Zhu P M, et a1. 3-D numerical simulation and dispersion analysis of in-seam wave in underground coalmine[J]. Chinese J. Geophys. (in Chinese), 2012, 55(2): 645 654.

[3] Yang Z, Ge M C, Wang S G. Characteristics of transmitting channel wave in a coal seam [J]. Mining Science \&Technology, 2009, 19: $331 \sim 336$.

[4] Zhu G M, Li G H, Cheng J Y. Numerical simulation of seismic survey in coal mine roadway[J]. Journal of China Coal Society[J], 2008, 33(11): $1263 \sim 1267$. 